\title{
DERIVATIONS OF LIE ALGEBRAS ${ }^{1}$
}

\author{
BY SHIGEAKI TÔGÔ
}

\author{
Communicated by S. Smale, March 24, 1966
}

1. It is known as a theorem of E. Schenkman and N. Jacobson that every nilpotent Lie algebra over a field of arbitrary characteristic has an outer derivation (see [1]). In connection with this theorem, we know the following two types of results, one showing a wider class of Lie algebras which have outer derivations and the other showing the existence of outer derivations in proper ideals of the derivation algebras. Namely, G. Leger [2] has shown that, if a Lie algebra over a field of characteristic 0 whose center is $\neq(0)$ has no outer derivations, it is not solvable and its radical is nilpotent. On the other hand, T. Satô [3] has shown that every nilpotent Lie algebra over a field of characteristic 0 has an outer derivation in the radical of its derivation algebra. We shall generalize and sharpen these results and give more detailed results on outer derivations of Lie algebras over a field of arbitrary characteristic.

2. We denote by $Z(H)$ the center of a Lie algebra $H$. Then we have

THEOREM 1. Every Lie algebra $L$ over a field $\Phi$ of arbitrary characteristic such that $L \neq L^{2}$ and $Z(L) \neq(0)$ has an outer derivation. More precisely, such a Lie algebra $L$ has a nilpotent outer derivation $D$ such that $D^{2}=0$, unless $L$ is either 1-dimensional or the direct sum of a 1-dimensional ideal and of an ideal $L_{1}$ such that $L_{1}=L_{1}^{2}$ and $Z\left(L_{1}\right)=(0)$.

In the case where $L$ is not abelian and has no abelian direct summands, take a subspace $M$ of $L$ of codimension 1 containing $L^{2}$. Then $M$ is an ideal of $L$ and $[L, Z(M)] \neq Z(M)$. Choose an element $e$ of $L$ such that $L=\Phi e+M$ and an element $z$ of $Z(M)$ which is not in $[L, Z(M)]$. Then the endomorphism $D$ of $L$ defined in such a way that $D e=z$ and $D M=(0)$ is an outer derivation of $L$ such that $D^{2}=0$.

CoRollary. Let $L$ be a Lie algebra over a field of characteristic 0 such that $Z(L) \neq(0)$ and $R$ be the radical of $L$. If $L$ has no outer derivations, $L$ is not solvable and $R=[L, R]$.

There is another class of nonsolvable Lie algebras which have outer derivations. Namely:

\footnotetext{
${ }^{1}$ Research supported in part by the National Science Foundation, grant number GP-3990.
} 
THEOREM 2. Let $L$ be a nonsolvable Lie algebra over a field of characteristic 0 and $R$ be the radical of $L$. If $R$ has a semisimple outer derivation in the radical of its derivation algebra, then $L$ has a semisimple outer derivation.

This can be shown by using the following fact: Let $L=S+R$ be a Levi decomposition of $L$. Then among maximal toroidal subalgebras of the radical of the derivation algebra of $R$, there exists one which can be imbedded in the set of all derivations of $L$ which map $S$ into (0). A consequence of the theorem is that if $R$ is nilpotent and has a derivation whose trace is $\neq 0$ then $L$ has a semisimple outer derivation.

3. We shall call a Lie algebra $L$ over a field $\Phi$ to be of type $(T)$ provided

$$
L=\Phi e_{1}+\Phi e_{1^{\prime}}+\cdots+\Phi e_{n}+\Phi e_{n^{\prime}}+L^{2}
$$

where

$$
\left[e_{j}, e_{k}\right]=\left[e_{j^{\prime}}, e_{k^{\prime}}\right]=0, \quad\left[e_{j}, e_{k^{\prime}}\right]=\delta_{j k} z \text { with } \quad 0 \neq z \in Z(L),
$$

and

$$
\left[e_{j}, L^{2}\right]=\left[e_{j^{\prime}}, L^{2}\right]=(0) \quad \text { for } j, k=1,2, \cdots, n .
$$

We denote by $\mathfrak{D}(L)$ the derivation algebra of a Lie algebra $L$, by $\Re$ the radical of $\mathfrak{D}(L)$, by $\mathfrak{R}_{0}$ the abelian ideal of $\mathfrak{D}(L)$ consisting of all derivations which map $L$ into $L^{2}$ and $L^{2}$ in to (0), by $\mathbb{S}(L)$ the ideal of $\mathfrak{D}(L)$ consisting of all central derivations, and by $\mathfrak{S}_{0}$ the abelian ideal of $\mathfrak{D}(L)$ consisting of all central derivations which map $Z(L)$ into (0). Then we have

THEOREM 3. Let $L$ be a Lie algebra over a field of arbitrary characteristic such that $L \neq L^{2}$ and $Z(L) \neq(0)$.

(1) If $L$ is not abelian and has no abelian direct summands and if $L$ is not of type $(\mathrm{T})$, then $L$ has an outer derivation in $\mathfrak{R}_{0}$.

(2) Assume that $L$ is not abelian but has an abelian direct summand. If $Z(L)$ is not a direct summand of $L$, then $L$ has an outer derivation in $\mathfrak{R}_{0} \cap \mathfrak{E}(L)$. If $Z(L)$ is a direct summand of $L$ and $L / Z(L)$ does not coincide with the derived algebra, then $L$ has an outer derivation in $\mathbb{E}_{0}$. If $Z(L)$ is a direct summand of $L$ and $L / Z(L)$ coincides with the derived algebra, then $L$ has a semisimple outer derivation in $\Re$.

(3) If $L$ is either abelian or a Lie algebra of type (T) such that $L^{(1)} \neq L^{(2)}$, then $L$ has a semisimple outer derivation in $\Re$.

In the above statements, $\mathfrak{R}_{0}, \mathfrak{N}_{0} \cap \mathfrak{S}(L), \mathfrak{S}_{0}$ and $\Re$ cannot be replaced by any smaller ideals of $\mathfrak{D}(L)$. 
This can be shown by using the following fact: $L$ is of type $(T)$ if

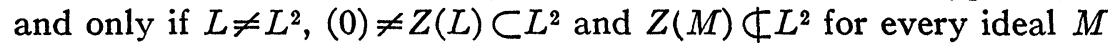
of $L$ of codimension 1 .

COROLlaRy 1. Let $L$ be a Lie algebra over a field of arbitrary characteristic such that $L \neq L^{(1)}, L^{(1)} \neq L^{(2)}$ and $Z(L) \neq(0)$. Then $L$ has an outer derivation in $\Re$.

A solvable Lie algebra $L$ of type $(T)$ is such that $L^{2}=\Phi z$ and therefore $L$ is nilpotent and $L^{(1)} \neq L^{(2)}$. Hence by Theorem 3 we have

COROLLARY 2. Every solvable Lie algebra $L$ over a field of arbitrary characteristic such that $Z(L) \neq(0)$ has an outer derivation in $\Re$. More precisely, if $L$ is not abelian and not of type $(T)$, then $L$ has an outer derivation in $\mathfrak{R}_{0}$ unless $Z(L)$ is a direct summand of $L$.

4. Let $L$ be a Lie algebra over a field of characteristic 0 . Denote by $A(L)$ the group of all automorphisms of $L$ and by $A_{0}(L)$ the irreducible component of $A(L)$. We shall call $\sigma \in A(L)$ to be outer provided it does not belong to the smallest algebraic subgroup of $A(L)$ whose Lie algebra contains all inner derivations. Then an application of Theorem 1 is the following:

THEOREM 4. Let $L$ be a Lie algebra over a field of characteristic 0 . If $L \neq L^{2}, Z(L) \neq(0)$ and the Lie algebra of all inner derivations of $L$ is algebraic, then $L$ has an outer automorphism in $A_{0}(L)$.

\section{REFERENCES}

1. N. Jacobson, $A$ note on automorphisms and derivations of Lie algebras, Proc. Amer. Math. Soc. 6 (1955), 281-283.

2. G. Leger, Derivations of Lie algebras. III, Duke Math. J. 30 (1963), 637-645.

3. T. Satô, On derivations of nilpotent Lie algebras, Tôhoku Math. J. 17 (1965), 244-249.

University of California, Berkeley and HIROSHIMA UNIVERSITY 